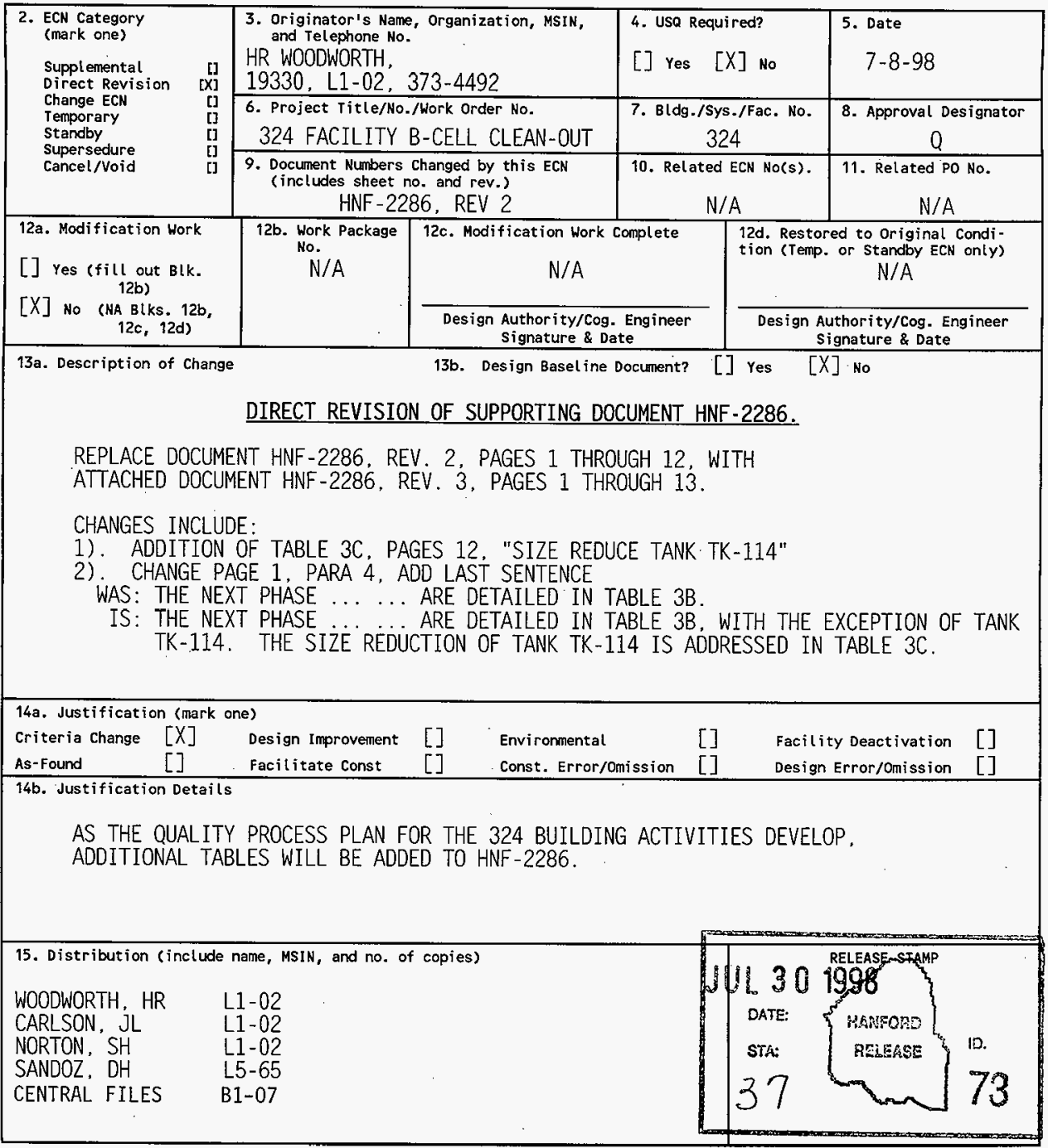




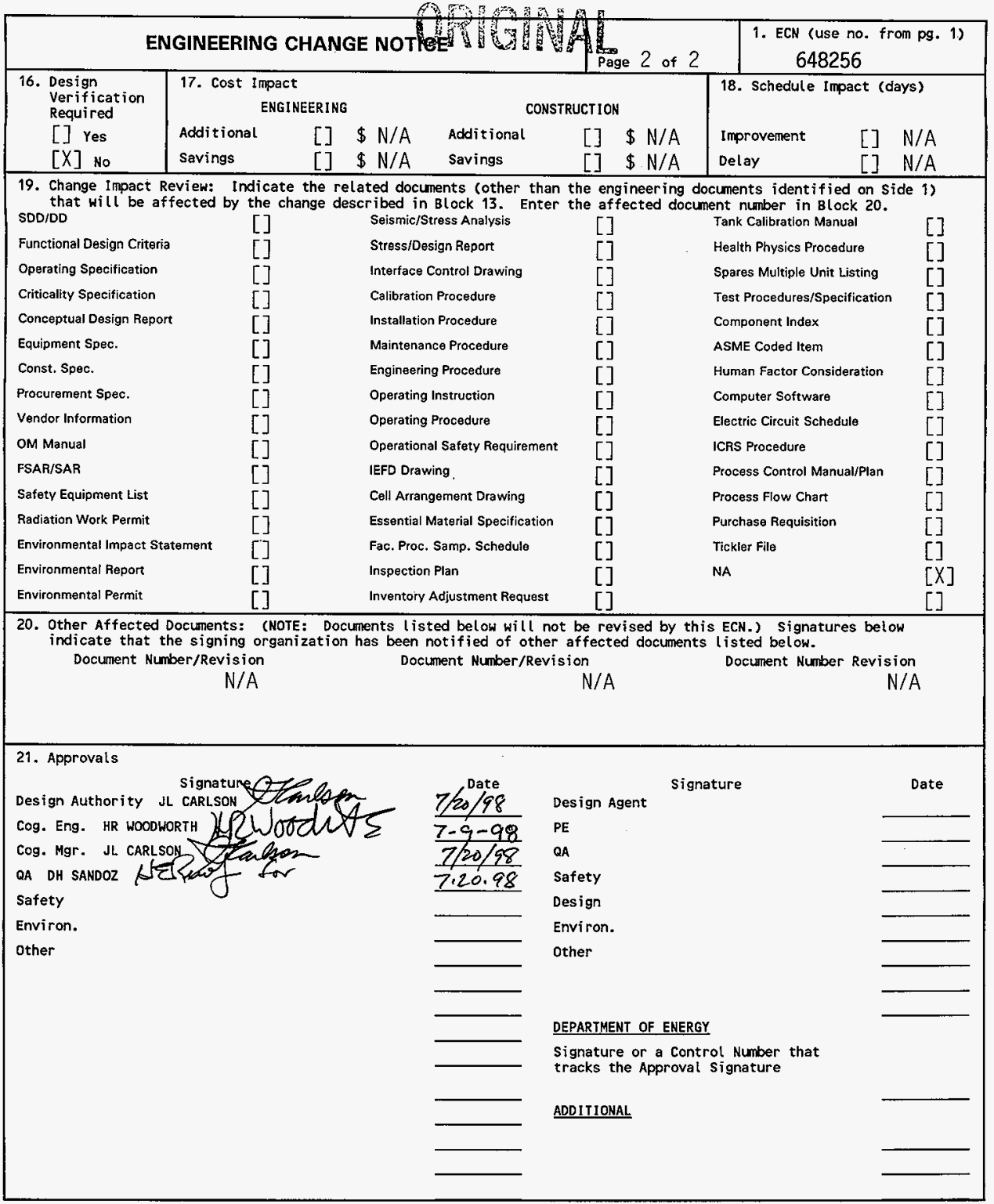


HNF-2286, Rev. 3

\section{Facility B-Cell Quality Process Plan}

J.L. Carison

BWHC, Richiand, WA 99352

U.S. Department of Energy Contract DE-AC06-96RL13200

EDT/ECN: 648253

Org Code: 19330

B\&R Code: EX7050000

UC: UC-506

Charge Code: K4BAA

Tota1 Pages: 15

HAW 98300

Key Words: B-Cell, Quality Process Plan, Hot-Work

Abstract: Quality Process Plan for the Restart of Cel1 Hot-Work. Addition of Table 3 C.

TRADEMARK DISCLAIMER. Reference herein to any specific comercial product, process, or service by trade name, trademark, manufacturer, or otherwise, does not necessarily constitute or imply its endorsement, recommendation, or favoring by the United States Government or any agency thereof or its contractors or subcontractors.

Printed in the United States of America. To obtain copies of this document, contact: Document Control Services, P.0. Box 950, Mailstop H6-08, Richland WA 99352, Phone (509) 372-2420;

Fax (509) 376-4989.
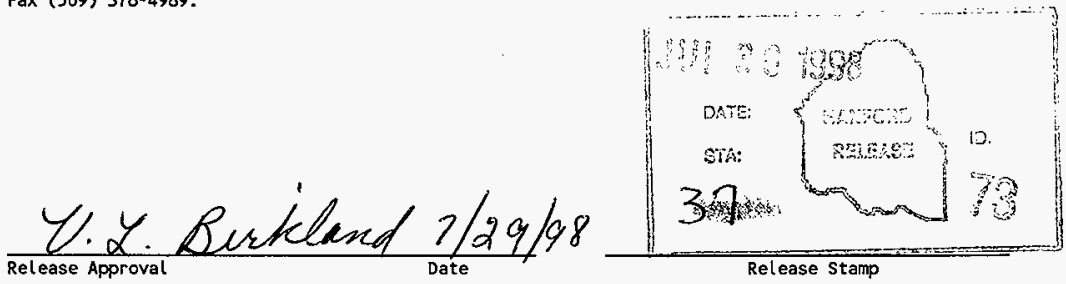

Release Stamp

\section{Approved for Public Release}


(2) Title

324 Facility B-Cell Quality Process Plan

CHANGE CONTROL RECORD

\begin{tabular}{l|ll} 
(3) Revision & (4) Description of Change - Replace, Add, and Delete Pages
\end{tabular} Rev. 0 (7) INITIAL ISSUE, EDT 623063, 03-13-1998

Rev. 1 DIRECT REVISION, ADDITION OF TABLE 2. MINOR TEXT CHANGES TABLE 1. PER ECN-647560

REV. 2

DIRECT REVISION, MINOR TEXT CHANGES AND THE ADDITION OF TABLE 3A AND 3B, PER ECN 648254

REV. $\beta S$
DIRECT REVISION. ADDITION OF TABLE 3C, PER ECN 648256
Authorized for Release

(5) Cog. Engr. (6) Cog. Mgr. Date $\mathrm{N} / \mathrm{A}$ $N / A$

JL CARLSON

$\mathrm{HR}$

WOODWORTH

$3 / 16 / 98$

$\mathrm{HR}$

WOODWORTH

$3 L$ CARLSON

$6 / 8 / 98$

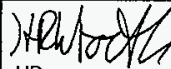

$H R$

WOODWORTH

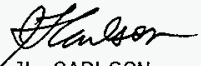

JL CARLSON 7/8/98 


\section{Facility B-Cell Quality Process Plan \\ HNF-2286 (Rev. 3)}

B-Cell is currently being cleaned out (i.e., removal of equipment, fixtures and residual radioactive materials) and deactivated. TPA Milestone M-89-02 dictates that all mixed waste and equipment be removed from B-Cell by $5 / 31 / 99$. The following sections describe the major activities that remain for completion of the TPA milestone.

\section{Size Reduce Tank 119 and Misc. Equipment}

This activity is the restart of hotwork in B-Cell to size reduce the remainder of Tank 119 and other misc. pieces of equipment into sizes that can be loaded into a grout container. This activity also includes the process of preparing the containers for shipment from the cell. The specific activities and procedures used are detailed in Table 1.

\section{Load and Ship Low-Level Waste}

This activity covers the process of taking a grouted LLW container from B-Cell and loading it into the 3-82B cask in the REC airlock and Cask Handling Area (CHA) for shipment to the LLBG. The detailed activities and procedures for this part of cell cleanout are included in Table 2.

\section{Remove and Size Reduce the 1B Rack}

The next phase of cell cleanout is the removal of the $1 \mathrm{~B}$ rack from the cell. This includes the remaining activities to remove the rack from the cell wall and the written plan for cutting the rack apart. Specific work instructions detail the steps used to dismantle the rack to ensure that rigging concerns have been addressed satisfactorily. The plan also details how the tanks in the rack structure will be segmented and how heels in the tanks will be handled as mixed waste. The detailed activities and procedures for the removal of the $1 \mathrm{~B}$ rack is contained in Table 3a. Rack size reduction plans are detailed in Table $3 \mathrm{~b}$, with the exception of tank TK-114. The size reduction of tank TK-114 is addressed in Table $3 \mathrm{c}$.

\section{Collect Dispersible Material from Cell Floor}

This part of cell cleanout is the collection of dispersible material from the cell floor. The bulk of the cesium contamination in the cell is expected to be found in the caked material on the floor under the footprints of the racks. Part of this material will be picked up after the $1 \mathrm{~B}$ rack size reduction, part after the size reduction of the $2 \mathrm{~A}$ rack, and a final increment after the $1 \mathrm{~A}$ rack size reduction is completed. The process will cleanout the drainage trench along the east wall of the cell and the sump in the northeast corner of the floor. The primary methods that will be used are scraping the material into piles, 


\section{Facility B-Ce11 Quality Process Plan HNF-2286 (Rev. 3)}

picking up the piles with a clamshell or vacuum, then placement of the material in containers for later shipment to the PUREX tunnels as mixed waste. The detailed activities and procedures for this part of cell cleanout will be included in a table added to this document in the future.

\section{Remove and Size Reduce the 2A Rack}

This activity will be very similar to the $1 \mathrm{~B}$ rack size reduction. It will cover the work required to remove the rack from the wall and a specific plan for dismantlement of this rack to account for any possible rigging concerns. The tanks in the $2 \mathrm{~A}$ rack are expected to have minimum heals as these tanks were condensate receiver vessels. The detailed activities and procedures for this part of cell cleanout will be included in a table added to this document in the future.

\section{Size Reduce the 1A Rack}

This phase of the project will size reduce the $1 \mathrm{~A}$ rack. This rack has already been disconnected and pulled from the wall in to the cell. The specific plan for the reduction of this rack will include the very specific way the tanks in the rack will be dismantled to contain the internal parts of the tanks. This rack contains the process evaporator, tank TK-113, which is expected to contain a potential holdup of $1.5 \mathrm{M}$ curies of heel material. The size reduction planning will also address the dismantlement sequence to ensure all rigging concerns are addressed. The detailed activities and procedures for this part of cell cleanout will be included in a table added to this document in the future.

\section{Load and Ship Mixed Waste to PUREX Tunnels}

This part of the cleanout process involves the loading of the 22.5 Ton casks with the dispersible material and tank heels collected throughout the cell cleanout project. The casks will be loaded in the REC airlock and shipped to the PUREX plant by truck. The casks will be moved on to flatcars and the fully loaded railcars will be pushed into Tunnel \#2. The detailed activities and procedures for this part of cell cleanout will be included in a table added to this document in the future.

\section{Move Spent Fuel to A-Cell}

Part of the cell inventory of equipment are seven commercial fuel bundles stored along the west wall of the cell. When the remaining FRG equipment has been removed from A-Cell the bundles will be moved through the REC airlock to A-Cell. The process will place the bundles into new thimbles supported by a new storage rack. The old thimbles and storage racks in B-Cell will be size reduced and disposed of as LLW. The detailed 


\section{Facility B-Cell Quality Process P7an HNF-2286 (Rev. 3)}

activities and procedures for this part of cell cleanout will be included in a table added to this document in the future. 
324 Facility B-Cell Quality Process Plan

HNF-2286 (Rev. 3)

\begin{tabular}{|c|c|c|c|c|c|}
\hline \multicolumn{6}{|c|}{ Table 1- Size Reduce Tank 119 and Misc. Equipment } \\
\hline Activity & Product & $\begin{array}{l}\text { Work } \\
\text { Control } \\
\text { Number } \\
\text { (JCS No.) }\end{array}$ & $\begin{array}{l}\text { Applicable } \\
\text { Procedure and } \\
\text { Instructions }\end{array}$ & $\begin{array}{l}\text { Responsible } \\
\text { (Lead) } \\
\text { Individual }\end{array}$ & $\begin{array}{l}\text { Quality } \\
\text { Requirements }\end{array}$ \\
\hline $\begin{array}{l}\text { Verify } \\
\text { combustible } \\
\text { loading within } \\
\text { limits for cell }\end{array}$ & $\begin{array}{l}\text { Inventory } \\
\text { within limits }\end{array}$ & N/A & $\begin{array}{l}\text { 3I-SOP-REC-K-22 } \\
\text { 3I-SOP-REC-G-22 }\end{array}$ & $\begin{array}{l}\text { BWHC Shift } \\
\text { Sup. }\end{array}$ & $\begin{array}{l}\text { Procedure } \\
\text { approval } \\
\text { BWHC Shift } \\
\text { Sup. monthly } \\
\text { review } \\
\text { Fire Protection } \\
\text { Eng. monthly } \\
\text { review }\end{array}$ \\
\hline $\begin{array}{l}\text { Obtain } \\
\text { Hotwork } \\
\text { Permit }\end{array}$ & $\begin{array}{l}\text { Approved } \\
\text { permit }\end{array}$ & N/A & $\begin{array}{l}\text { 3I-SOP-REC-K-22 } \\
\text { HNF-PRO-356 }\end{array}$ & $\begin{array}{l}\text { BWHC Shift } \\
\text { Sup. }\end{array}$ & $\begin{array}{l}\text { Procedure step } \\
\text { Fire Protection } \\
\text { Eng. Approval } \\
\text { Facility mgr. } \\
\text { approval }\end{array}$ \\
\hline $\begin{array}{l}\text { Perform size } \\
\text { reduction of } \\
\text { Tank } 119 \\
\text { and misc. cell } \\
\text { debris }\end{array}$ & $\begin{array}{l}\text { Metal for } \\
\text { addition to } \\
\text { GC }\end{array}$ & N/A & $\begin{array}{l}\text { 31-SOP-REC-K-22 } \\
\text { 31-SOP-REC-A-38 } \\
\text { 31-SOP-REC-F-2 }\end{array}$ & $\begin{array}{l}\text { BWHC Shift } \\
\text { Sup. }\end{array}$ & $\begin{array}{l}\text { Procedure } \\
\text { steps } \\
\text { Procedure } \\
\text { steps } \\
\text { Procedure } \\
\text { steps }\end{array}$ \\
\hline
\end{tabular}


324 Facility B-Cell Quality Process Plan

HNF-2286 (Rev. 3)

\begin{tabular}{|c|c|c|c|c|c|}
\hline Activity & Product & $\begin{array}{l}\text { Work } \\
\text { Control } \\
\text { Number } \\
\text { (JCS No.) }\end{array}$ & $\begin{array}{l}\text { Applicable } \\
\text { Procedure and } \\
\text { Instructions }\end{array}$ & $\begin{array}{l}\text { Responsible } \\
\text { (Lead) } \\
\text { Individual }\end{array}$ & $\begin{array}{l}\text { Quality } \\
\text { Requirements }\end{array}$ \\
\hline $\begin{array}{l}\text { Rinse metal } \\
\text { piece }\end{array}$ & $\begin{array}{l}\text { Metal } \\
\text { cleaned for } \\
\text { addition to } \\
\text { GC }\end{array}$ & N/A & $\begin{array}{l}\text { 3I-SOP-REC-A-49 } \\
\text { 3I-SOP-REC-A-38 }\end{array}$ & $\begin{array}{l}\text { BWHC Shift } \\
\text { Sup. }\end{array}$ & $\begin{array}{l}\text { Procedure } \\
\text { approval } \\
\text { Waste coord. } \\
\text { approval } \\
\text { Procedure } \\
\text { steps }\end{array}$ \\
\hline Load GC & Filled GC & $N / A$ & $\begin{array}{l}\text { 3I-SOP-REC-A-49 } \\
\text { 3I-SOP-REC-A-38 }\end{array}$ & $\begin{array}{l}\text { BWHC Shift } \\
\text { Sup. }\end{array}$ & $\begin{array}{l}\text { Procedure } \\
\text { steps }\end{array}$ \\
\hline Weigh GC & $\begin{array}{l}\text { GC within } \\
\text { weight } \\
\text { limits }\end{array}$ & N/A & 3!-SOP-REC-A-49 & $\begin{array}{l}\text { BWHC Shift } \\
\text { Sup. }\end{array}$ & $\begin{array}{l}\text { Calibrated load } \\
\text { cell }\end{array}$ \\
\hline $\begin{array}{l}\text { Pre-Grout } \\
\text { dose profile }\end{array}$ & $\begin{array}{l}\text { GC within } \\
\text { dose limits }\end{array}$ & N/A & 3I-SOP-REC-A-49 & $\begin{array}{l}\text { BWHC Shift } \\
\text { Sup. }\end{array}$ & $\begin{array}{l}\text { Calibrated R-07 } \\
\text { RCT review }\end{array}$ \\
\hline $\begin{array}{l}\text { Approval to } \\
\text { grout }\end{array}$ & $\begin{array}{l}\text { GC ready } \\
\text { to grout }\end{array}$ & N/A & 3!-SOP-REC-A-49 & $\begin{array}{l}\text { BWHC Shift } \\
\text { Sup. }\end{array}$ & Procedure step \\
\hline $\begin{array}{l}\text { Grout } \\
\text { container }\end{array}$ & $\begin{array}{l}\text { GC ready } \\
\text { for final } \\
\text { dose profile }\end{array}$ & N/A & $\begin{array}{l}\text { 3I-SOP-REC-A-49 } \\
\text { 31-SOP-REC-A-65 }\end{array}$ & $\begin{array}{l}\text { BWHC Shift } \\
\text { Sup. }\end{array}$ & $\begin{array}{l}\text { Calibrated load } \\
\text { cell } \\
\text { Procedure } \\
\text { steps }\end{array}$ \\
\hline $\begin{array}{l}\text { Post-Grout } \\
\text { dose profile }\end{array}$ & $\begin{array}{l}\text { GC ready } \\
\text { for } \\
\text { shipment }\end{array}$ & N/A & 31-SOP-REC-A-49 & $\begin{array}{l}\text { BWHC Shift } \\
\text { Sup. }\end{array}$ & $\begin{array}{l}\text { Calibrated R-07 } \\
\text { RCT review }\end{array}$ \\
\hline
\end{tabular}


324 Facility B-Cell Quality Process PTan

HNF-2286 (Rev. 3)

\begin{tabular}{|c|c|c|c|c|c|}
\hline \multicolumn{6}{|c|}{ Table 2 - Load and Ship Low 119 Misc. Equipment } \\
\hline Activity & Product & $\begin{array}{l}\text { Work } \\
\text { Control } \\
\text { Number } \\
\text { (JCS No.) }\end{array}$ & $\begin{array}{l}\text { Applicable } \\
\text { Procedure and } \\
\text { Instructions }\end{array}$ & $\begin{array}{l}\text { Responsible } \\
\text { (Lead) } \\
\text { Individual }\end{array}$ & $\begin{array}{l}\text { Quality } \\
\text { Requirements }\end{array}$ \\
\hline Receive cask & $\begin{array}{l}\text { Facility } \\
\text { acceptance }\end{array}$ & N/A & 3I-SOP-REC-A-44 & $\begin{array}{l}\text { BWHC Shift } \\
\text { Sup. }\end{array}$ & $\begin{array}{l}\text { Procedure } \\
\text { approval by QA } \\
\text { and Packaging } \\
\text { Procedure } \\
\text { steps }\end{array}$ \\
\hline $\begin{array}{l}\text { Unload cask } \\
\text { from trailer }\end{array}$ & $\begin{array}{l}\text { Cask ready } \\
\text { to load }\end{array}$ & N/A & 3I-SOP-REC-A-44 & $\begin{array}{l}\text { BWHC Shift } \\
\text { Sup. }\end{array}$ & $\begin{array}{l}\text { Procedure } \\
\text { steps }\end{array}$ \\
\hline $\begin{array}{l}\text { Inspect cask } \\
\text { system }\end{array}$ & $\begin{array}{l}\text { Verify no } \\
\text { internal } \\
\text { debris }\end{array}$ & N/A & 3I-SOP-REC-A-42 & $\begin{array}{l}\text { BWHC Shift } \\
\text { Sup. }\end{array}$ & $\begin{array}{l}\text { Procedure } \\
\text { approval by QA } \\
\text { and Packaging } \\
\text { Procedure hold } \\
\text { point }\end{array}$ \\
\hline $\begin{array}{l}\text { Inspect } \\
\text { Hittman liner }\end{array}$ & $\begin{array}{l}\text { Verify no } \\
\text { damage }\end{array}$ & N/A & 3I-SOP-REC-A-42 & $\begin{array}{l}\text { BWHC Shift } \\
\text { Sup. }\end{array}$ & $\begin{array}{l}\text { Procedure hold } \\
\text { point }\end{array}$ \\
\hline $\begin{array}{l}\text { Place } \\
\text { Hittman liner } \\
\text { in } A L L\end{array}$ & $\begin{array}{l}\text { Liner ready } \\
\text { for GC }\end{array}$ & N/A & 3I-SOP-REC-A-42 & $\begin{array}{l}\text { BWHC Shift } \\
\text { Sup. }\end{array}$ & Procedure step \\
\hline $\begin{array}{l}\text { Move cask } \\
\text { into } A / L\end{array}$ & $\begin{array}{l}\text { Cask ready } \\
\text { for liner }\end{array}$ & N/A & 3I-SOP-REC-A-42 & $\begin{array}{l}\text { BWHC Shift } \\
\text { Sup. }\end{array}$ & Procedure step \\
\hline $\begin{array}{l}\text { Record GC } \\
\text { ID number }\end{array}$ & $\begin{array}{l}\text { Verify } \\
\text { waste } \\
\text { package }\end{array}$ & N/A & 3I-SOP-REC-A-42 & $\begin{array}{l}\text { BWHC Shift } \\
\text { Sup. }\end{array}$ & $\begin{array}{l}\text { Procedure hold } \\
\text { point }\end{array}$ \\
\hline
\end{tabular}


324 Facility B-Cell Quality Process Plan

HNF-2286 (Rev. 3)

Table 2 - Load and Ship Low 119 Misc. Equipment

\begin{tabular}{|c|c|c|c|c|c|}
\hline Activity & Product & $\begin{array}{l}\text { Work } \\
\text { Control } \\
\text { Number } \\
\text { (JCS No.) }\end{array}$ & $\begin{array}{l}\text { Applicable } \\
\text { Procedure and } \\
\text { Instructions }\end{array}$ & $\begin{array}{l}\text { Responsible } \\
\text { (Lead) } \\
\text { Individual }\end{array}$ & $\begin{array}{l}\text { Quality } \\
\text { Requirements }\end{array}$ \\
\hline $\begin{array}{l}\text { Load Hittman } \\
\text { liner }\end{array}$ & $\begin{array}{l}\text { Liner ready } \\
\text { for lid }\end{array}$ & $N / A$ & 3I-SOP-REC-A-42 & $\begin{array}{l}\text { BWHC Shift } \\
\text { Sup. }\end{array}$ & Procedure step \\
\hline $\begin{array}{l}\text { Load liner } \\
\text { into cask }\end{array}$ & $\begin{array}{l}\text { Cask ready } \\
\text { for removal } \\
\text { from } A / L\end{array}$ & & 3I-SOP-REC-A-42 & $\begin{array}{l}\text { BWHC Shift } \\
\text { Sup. }\end{array}$ & Procedure step \\
\hline $\begin{array}{l}\text { Remove cask } \\
\text { from } A / L\end{array}$ & $\begin{array}{l}\text { Cask ready } \\
\text { for lid }\end{array}$ & $N / A$ & 3I-SOP-REC-A-42 & $\begin{array}{l}\text { BWHC Shift } \\
\text { Sup. }\end{array}$ & Procedure step \\
\hline $\begin{array}{l}\text { Torque cask } \\
\text { lid nuts }\end{array}$ & $\begin{array}{l}\text { Cask lid } \\
\text { secured }\end{array}$ & N/A & 3I-SOP-REC-A-42 & $\begin{array}{l}\text { BWHC Shift } \\
\text { Sup. }\end{array}$ & $\begin{array}{l}\text { Procedure hold } \\
\text { point } \\
\text { Calibrated } \\
\text { torque } \\
\text { wrenches }\end{array}$ \\
\hline $\begin{array}{l}\text { Perform cask } \\
\text { leak test }\end{array}$ & $\begin{array}{l}\text { Verify cask } \\
\text { sealed }\end{array}$ & $N / A$ & 3I-SOP-REC-A-42 & $\begin{array}{l}\text { BWHC Shift } \\
\text { Sup. }\end{array}$ & $\begin{array}{l}\text { Procedure hold } \\
\text { point } \\
\text { Calibrated test } \\
\text { device }\end{array}$ \\
\hline $\begin{array}{l}\text { Load cask } \\
\text { onto trailer }\end{array}$ & $\begin{array}{l}\text { Cask ready } \\
\text { to ship }\end{array}$ & N/A & 31-SOP-REC-A-44 & $\begin{array}{l}\text { BWHC Shift } \\
\text { Sup. }\end{array}$ & $\begin{array}{l}\text { Procedure } \\
\text { steps }\end{array}$ \\
\hline $\begin{array}{l}\text { Review } \\
\text { loading and } \\
\text { shipping } \\
\text { records }\end{array}$ & $\begin{array}{l}\text { Verify } \\
\text { shipping } \\
\text { documents }\end{array}$ & N/A & 31-SOP-REC-A-44 & $Q C$ inspector & $\begin{array}{l}\text { QC Inpection } \\
\text { Plan } \\
\text { QC hold point }\end{array}$ \\
\hline Ship cask & $\begin{array}{l}\text { LLW } \\
\text { shipment }\end{array}$ & N/A & 3I-SOP-REC-A-44 & $\begin{array}{l}\text { BWHC Shift } \\
\text { Sup. }\end{array}$ & Procedure step \\
\hline
\end{tabular}


324 Facility B-Cell Quality Process Plan

HNF-2286 (Rev. 3)

\begin{tabular}{|c|c|c|c|c|c|}
\hline \multicolumn{6}{|c|}{ Table 3a - Remove the 1B Rack } \\
\hline Activity & Product & $\begin{array}{l}\text { Work } \\
\text { Control } \\
\text { Number } \\
\text { (JCS No.) } \\
\end{array}$ & $\begin{array}{l}\text { Applicable } \\
\text { Procedure and } \\
\text { Instructions }\end{array}$ & $\begin{array}{l}\text { Responsible } \\
\text { (Lead) } \\
\text { Individual }\end{array}$ & $\begin{array}{l}\text { Quality } \\
\text { Requirements }\end{array}$ \\
\hline $\begin{array}{l}\text { Prepare } \\
\text { Equip, Cell \& } \\
\text { Attach Winch }\end{array}$ & $\begin{array}{l}\text { Facilitate } \\
\text { Removal of } \\
\text { 1B Rack }\end{array}$ & $\begin{array}{l}31-98-00150 \\
\text { (Step 1-16) }\end{array}$ & 3I-SOP-REC-K- $30^{\star *}$ & $\begin{array}{l}\text { BWHC Shift } \\
\text { Sup. }\end{array}$ & $\begin{array}{l}\text { Work Package } \\
\text { Steps }\end{array}$ \\
\hline $\begin{array}{l}\text { Remove } \\
\text { MSMs at } \\
\text { South } \\
\text { Window }\end{array}$ & $\begin{array}{l}\text { Facilitate } \\
\text { Removal of } \\
\text { 1B Rack }\end{array}$ & $\begin{array}{l}31-98-00150 \\
\text { (Step 18) }\end{array}$ & 31-SOP-REC-K-02 ** & $\begin{array}{l}\text { BWHC Shift } \\
\text { Sup. }\end{array}$ & $\begin{array}{l}\text { Procedure and } \\
\text { Work Package } \\
\text { step }\end{array}$ \\
\hline $\begin{array}{l}\text { Remove 1B } \\
\text { Rack From } \\
\text { Wall } \\
\end{array}$ & $\begin{array}{l}\text { 1B Rack } \\
\text { Ready for } \\
\text { Reduction }\end{array}$ & $\begin{array}{l}31-98-00150 \\
\text { (Step 25) }\end{array}$ & N/A & $\begin{array}{l}\text { BWHC Shift } \\
\text { Sup. }\end{array}$ & $\begin{array}{l}\text { Work Package } \\
\text { Steps }\end{array}$ \\
\hline $\begin{array}{l}\text { Install MSMs } \\
\text { at South } \\
\text { Window }\end{array}$ & $\begin{array}{l}\text { Restor B-Cell } \\
\text { Capability }\end{array}$ & $\begin{array}{l}31-98-00150 \\
(\text { Step 28) }\end{array}$ & 3I-SOP-REC-K-02** & $\begin{array}{l}\text { BWHC Shift } \\
\text { Sup. }\end{array}$ & $\begin{array}{l}\text { Procedure and } \\
\text { Work Package } \\
\text { steps }\end{array}$ \\
\hline $\begin{array}{l}\text { Install Wall } \\
\text { Shield Plugs }\end{array}$ & $\begin{array}{l}\text { Provide } \\
\text { Shielding for } \\
\text { Airlock and } \\
\text { B-Cell }\end{array}$ & $\begin{array}{l}31-98-00150 \\
\text { (Step 32-35) }\end{array}$ & $\begin{array}{l}\text { 3I-SOP-REC-A-04** } \\
\text { 3I-SOP-REC-A-12**} \\
\text { 3I-SOP-REC-F-11**} \\
\text { 3I-SOP-REC-F-11A }\end{array}$ & $\begin{array}{l}\text { BWHC Shift } \\
\text { Sup. }\end{array}$ & $\begin{array}{l}\text { Procedure and } \\
\text { Work Package } \\
\text { steps }\end{array}$ \\
\hline
\end{tabular}


324 Facility B-Cell Quality Process Plan

HNF-2286 (Rev. 3)

\begin{tabular}{|c|c|c|c|c|c|}
\hline \multicolumn{6}{|c|}{ Table 3b - Size Reduce the 1B Rack } \\
\hline Activity & Product & $\begin{array}{l}\text { Work } \\
\text { Control } \\
\text { Number } \\
\text { (JCS No.) }\end{array}$ & $\begin{array}{l}\text { Applicable } \\
\text { Procedure and } \\
\text { Instructions }\end{array}$ & $\begin{array}{l}\text { Responsible } \\
\text { (Lead) } \\
\text { Individual }\end{array}$ & $\begin{array}{l}\text { Quality } \\
\text { Requirements }\end{array}$ \\
\hline $\begin{array}{l}\text { Remove Misc. } \\
\text { Piping and } \\
\text { Agitator }\end{array}$ & $\begin{array}{l}\text { Metal for } \\
\text { Addition } \\
\text { to GC }\end{array}$ & $\begin{array}{l}\text { 3I-98-00151 } \\
\text { (Step 3) }\end{array}$ & $\begin{array}{l}\text { 3I-SOP-REC-A-49* } \\
\text { 31-SOP-REC-K-22* } \\
\text { 3I-SOP-REC-A-38* } \\
\text { 3I-SOP-REC-F-02* } \\
\text { 3I-SOP-REC-K-05* }\end{array}$ & $\begin{array}{l}\text { BWHC Shift } \\
\text { Sup. }\end{array}$ & $\begin{array}{l}\text { Procedure and } \\
\text { Work package } \\
\text { Steps } \\
\text { Peer review } \\
\text { CG } \\
\text { calculations }\end{array}$ \\
\hline $\begin{array}{l}\text { Reduce } \\
\text { Connector } \\
\text { Face }\end{array}$ & $\begin{array}{l}\text { Metal for } \\
\text { Addition } \\
\text { to GC }\end{array}$ & $\begin{array}{l}\text { 3I-98-00151 } \\
\text { (Step 7) }\end{array}$ & $\begin{array}{l}\text { 3I-SOP-REC-A-49* } \\
\text { 3I-SOP-REC-K-22* } \\
\text { 3I-SOP-REC-A-38* } \\
\text { 3I-SOP-REC-F-02* } \\
\text { 3I-SOP-REC-K-05* }\end{array}$ & $\begin{array}{l}\text { BWHC Shift } \\
\text { Sup. }\end{array}$ & $\begin{array}{l}\text { Procedure and } \\
\text { Work package } \\
\text { Steps } \\
\text { Peer review } \\
\text { CG } \\
\text { calculations }\end{array}$ \\
\hline $\begin{array}{l}\text { Reduce Lower } \\
\text { Rack } \\
\text { Structure and } \\
\text { Plugs }\end{array}$ & $\begin{array}{l}\text { Metal for } \\
\text { Addition } \\
\text { to GC }\end{array}$ & $\begin{array}{l}\text { 31-98-00151 } \\
\text { (Step 9) }\end{array}$ & $\begin{array}{l}\text { 31-SOP-REC-A-49* } \\
\text { 31-SOP-REC-K-22* } \\
\text { 31-SOP-REC-A-38* } \\
\text { 31-SOP-REC-F-02* } \\
\text { 31-SOP-REC-K-05* }\end{array}$ & $\begin{array}{l}\text { BWHC Shift } \\
\text { Sup. }\end{array}$ & $\begin{array}{l}\text { Procedure and } \\
\text { Work package } \\
\text { Steps } \\
\text { Peer review } \\
\text { CG } \\
\text { calculations }\end{array}$ \\
\hline $\begin{array}{l}\text { Remove } \\
\text { E-112 } \\
\text { Reflux } \\
\text { Condenser }\end{array}$ & $\begin{array}{l}\text { Metal for } \\
\text { Addition } \\
\text { to GC }\end{array}$ & $\begin{array}{l}\text { 31-98-00151 } \\
\text { (Step 11) }\end{array}$ & $\begin{array}{l}\text { 3I-SOP-REC-A-49* } \\
\text { 3I-SOP-REC-K-22* } \\
\text { 3I-SOP-REC-A-38* } \\
\text { 3I-SOP-REC-F-02* } \\
\text { 3I-SOP-REC-K-05* }\end{array}$ & $\begin{array}{l}\text { BWHC Shift } \\
\text { Sup. }\end{array}$ & $\begin{array}{l}\text { Procedure and } \\
\text { Work package } \\
\text { Steps } \\
\text { Peer review } \\
\text { CG } \\
\text { calculations }\end{array}$ \\
\hline
\end{tabular}


324 Facility B-Cell Quality Process Plan

HNF-2286 (Rev. 3)

\begin{tabular}{|c|c|c|c|c|c|}
\hline \multicolumn{6}{|c|}{ Table 3b - Size Reduce the 1B Rack (cont.) } \\
\hline Activity & Product & $\begin{array}{l}\text { Work } \\
\text { Control } \\
\text { Number } \\
\text { (JCS No.) }\end{array}$ & $\begin{array}{l}\text { Applicable } \\
\text { Procedure and } \\
\text { Instructions }\end{array}$ & $\begin{array}{l}\text { Responsible } \\
\text { (Lead) } \\
\text { Individual }\end{array}$ & $\begin{array}{l}\text { Quality } \\
\text { Requirements }\end{array}$ \\
\hline $\begin{array}{l}\text { Reduce Tank } \\
\text { TK-112 }\end{array}$ & $\begin{array}{l}\text { Metal for } \\
\text { Addition } \\
\text { to GC }\end{array}$ & $\begin{array}{l}\text { 31-98-00151 } \\
\text { (Step 12) }\end{array}$ & $\begin{array}{l}\text { 3I-SOP-REC-A-49* } \\
\text { 3I-SOP-REC-K-22* } \\
\text { 3I-SOP-REC-A-38* } \\
\text { 3I-SOP-REC-F-02* } \\
\text { 3I-SOP-REC-K-05* }\end{array}$ & $\begin{array}{l}\text { BWHC Shift } \\
\text { Sup. }\end{array}$ & $\begin{array}{l}\text { Procedure and } \\
\text { Work package } \\
\text { Steps } \\
\text { Peer review } \\
\text { CG } \\
\text { calculations }\end{array}$ \\
\hline $\begin{array}{l}\text { Remove Tank } \\
\text { TK-114 }\end{array}$ & $\begin{array}{l}\text { Tank } \\
\text { TK-114 } \\
\text { set aside } \\
\text { for later } \\
\text { Disposal }\end{array}$ & $\begin{array}{l}\text { 3I-98-00151 } \\
\text { (Step 15) }\end{array}$ & $\begin{array}{l}\text { 3I-SOP-REC-A-49* } \\
\text { 3I-SOP-REC-K-22* } \\
\text { 3I-SOP-REC-A-38* } \\
\text { 3I-SOP-REC-F-02* } \\
\text { 3I-SOP-REC-K-05* }\end{array}$ & $\begin{array}{l}\text { BWHC Shift } \\
\text { Sup. }\end{array}$ & $\begin{array}{l}\text { Procedure and } \\
\text { Work package } \\
\text { Steps } \\
\text { Peer review } \\
\text { CG } \\
\text { calculations }\end{array}$ \\
\hline $\begin{array}{l}\text { Reduce Upper } \\
\text { Rack } \\
\text { Structure }\end{array}$ & $\begin{array}{l}\text { Metal for } \\
\text { Addition } \\
\text { to GC }\end{array}$ & $\begin{array}{l}31-98-00151 \\
\text { (Step 16) }\end{array}$ & $\begin{array}{l}\text { 31-SOP-REC-A-49* } \\
\text { 3I-SOP-REC-K-22* } \\
\text { 3l-SOP-REC-A-38* } \\
\text { 3I-SOP-REC-F-02* } \\
\text { 31-SOP-REC-K-05* }\end{array}$ & $\begin{array}{l}\text { BWHC Shift } \\
\text { Sup. }\end{array}$ & $\begin{array}{l}\text { Procedure and } \\
\text { Work package } \\
\text { Steps } \\
\text { Peer review } \\
\text { CG } \\
\text { calculations }\end{array}$ \\
\hline
\end{tabular}


324 Facility B-Cell Quality Process Plan

HNF-2286 (Rev. 3)

\begin{tabular}{|l|l|l|l|l|l|}
\hline \multicolumn{7}{|c|}{ Table 3b - Size Reduce the 1B Rack (cont.) } \\
\hline Activity & Product & $\begin{array}{l}\text { Work } \\
\text { Control } \\
\text { Number } \\
\text { (JCS No.) }\end{array}$ & $\begin{array}{l}\text { Applicable } \\
\text { Procedure and } \\
\text { Instructions }\end{array}$ & $\begin{array}{l}\text { Responsibl } \\
\text { e (Lead) } \\
\text { Individual }\end{array}$ & $\begin{array}{l}\text { Quality } \\
\text { Requirements }\end{array}$ \\
\hline $\begin{array}{l}\text { Reduce } \\
\text { Upper } \\
\text { Plugs }\end{array}$ & $\begin{array}{l}\text { Metal for } \\
\text { Addition } \\
\text { to GC }\end{array}$ & $\begin{array}{l}3 \text { G-98-00151 } \\
\text { (Step 18-19) }\end{array}$ & $\begin{array}{l}\text { 3l-SOP-REC-A-49* } \\
\text { 3l-SOP-REC-K-22* } \\
\text { 3l-SOP-REC-A-38* } \\
\text { 3l-SOP-REC-F-02* } \\
\text { 3l-SOP-REC-K-05* }\end{array}$ & $\begin{array}{l}\text { BWHC Shift } \\
\text { Sup. }\end{array}$ & $\begin{array}{l}\text { Procedure and } \\
\text { Work package } \\
\text { Steps }\end{array}$ \\
\hline
\end{tabular}




\section{Facility B-Ce11 Quality Process Plan \\ HNF-2286 (Rev. 3)}

\begin{tabular}{|l|l|l|l|l|l|}
\hline \multicolumn{7}{|c|}{ Table 3c-Size Reduce the 1B Rack (tank TK-114) } \\
\hline Activity & Product & $\begin{array}{l}\text { Work } \\
\text { Control } \\
\text { Number } \\
\text { (JCS No.) }\end{array}$ & $\begin{array}{l}\text { Applicable } \\
\text { Procedure and } \\
\text { Instructions }\end{array}$ & $\begin{array}{l}\text { Responsible } \\
\text { (Lead) } \\
\text { Individual }\end{array}$ & $\begin{array}{l}\text { Quality } \\
\text { Requirements }\end{array}$ \\
\hline $\begin{array}{l}\text { Reduce } \\
\text { Tank TK- } \\
114\end{array}$ & $\begin{array}{l}\text { Metal for } \\
\text { Addition } \\
\text { to GC }\end{array}$ & $\begin{array}{l}\text { 3I-98-00151 } \\
\text { (Step 15.1 } \\
\text { WCN-03) }\end{array}$ & $\begin{array}{l}\text { 3l-SOP-REC-A-49* } \\
\text { 3l-SOP-REC-K-22* } \\
\text { 3I-SOP-REC-A-38* } \\
\text { 3I-SOP-REC-F-02* } \\
\text { 3I-SOP-REC-K-05* }\end{array}$ & $\begin{array}{l}\text { BWHC Shift } \\
\text { Sup. }\end{array}$ & $\begin{array}{l}\text { Procedure and } \\
\text { Work package } \\
\text { Steps }\end{array}$ \\
\hline
\end{tabular}

** Common Facility Procedures, not provided for Review

3I-SOP-REC-K-30, Installation/Removal of services Via Split Plugs and Operating Transfer Ports

3I-SOP-REC-K-02, Maniuplator Removal and Installation

31-SOP-REC-A-04, Airlock/Cell Access

31-SOP-REC-A-12, Hot Cell Shielding Door Controls

3I-SOP-REC-F-11, Dress/Undress Procedure for Fresh Air Hood

3|-SOP-REC-F-11A, Dress/Undress Procedure for Full Face Air Purifying Respirator and Two Pair of PCs

*Procedures Previousiy Reviewed

Reviewed for Table 1

3!-SOP-REC-A-49, Preparing/Loading Low Level Waste (LLW) Grout Containers

3i-SOP-REC-K-22, In Cell Operation of thermal Arc Plasma Cutting System

3I-SOP-REC-A-38, Medium Pressure Spray Decontamination

3I-SOP-REC-F-02, Fire Safety Guidelines for Radiochemical Hot Cells

3I-SOP-REC-K-05, Radiochemical Engineering Cells (REC) Cranes

3I-SOP-REC-G-22, Hot Cell Combustible Materials Inventory 


\section{Facility B-Cell Quality Process Plan}

HNF-2286 (Rev. 3)

Tables to be Added by Document Revision

Table 4 - Collect Dispersible Material from Cell Floor

Table 5 - Remove and Size Reduce the 2A Rack

Table 6 - Size Reduce the 1A Rack

Table 7 - Load and Ship Mixed Waste to PUREX Tunnels

Table 8 - Move Spent Fuel to A-Cell 